\title{
Samarium oxide as a radiotracer to evaluate the in vivo biodistribution of PLGA nanoparticles
}

\author{
Vusani Mandiwana ${ }^{\mathrm{a}, \mathrm{d}, *}$, Lonji Kalombo ${ }^{\mathrm{a}}$, Kobus Venter ${ }^{\mathrm{b}}$, Mike Sathekge ${ }^{\mathrm{c}}$, Anne Grobler ${ }^{\mathrm{d}}$, Jan \\ Rijn Zeevaart ${ }^{\mathrm{d}, \mathrm{e}}$ \\ ${ }^{a}$ Centre of Polymers and Composites, CSIR, PO Box 395, Pretoria, 0001, South Africa; E-mails: \\ VMandiwana@csir.co.za; LKalombo@csir.co.za \\ ${ }^{\mathrm{b}}$ South African Medical Research Council, Private Bag X385, Pretoria, 0001, South Africa; E-mail: \\ Kobus.Venter@mrc.ac.za \\ ${ }^{\mathrm{c}}$ Department of Nuclear Medicine, University of Pretoria and Steve Biko Academic Hospital, Private Bag \\ X169, Pretoria, 0001, South Africa; E-mail: Mike.Sathekge@up.ac.za \\ ${ }^{\mathrm{d}}$ DST/NWU Preclinical drug development platform, North-West University, Private Bag X6001, \\ Potchefstroom, 2520, South Africa; \\ E-mail: Anne.Grobler@nwu.ac.za \\ ${ }^{\mathrm{e}}$ Radiochemistry, South African Nuclear Energy Corporation, Pelindaba, PO Box 582, Pretoria, 0001, South \\ Africa; \\ E-mail: janrijn.zeevaart@necsa.co.za
}

*Author to whom correspondence should be addressed; Centre of Polymers and Composites, CSIR, PO Box 395, Pretoria, 0001, South Africa; E-mail: VMandiwana@csir.co.za; Tel.: +27 12841 2985;

Fax: +27866105619

\begin{abstract}
Developing nanoparticulate delivery systems that will allow easy movement and localisation of a drug to the target tissue and provide more controlled release of the drug in vivo is a challenge in nanomedicine. The aim of this study was to evaluate the biodistribution of poly (D,L-lactide-coglycolide) (PLGA) nanoparticles containing samarium-153 oxide $\left(\left[{ }^{153} \mathrm{Sm}\right] \mathrm{Sm}_{2} \mathrm{O}_{3}\right)$ in vivo to prove that orally administered nanoparticles alter the biodistribution of a drug.

These were then activated in a nuclear reactor to produce radioactive ${ }^{153} \mathrm{Sm}$-loaded -PLGA nanoparticles. The nanoparticles were characterized for size, Zeta potential and morphology. The nanoparticles were orally and intravenously (IV) administered to rats in order to trace their uptake through imaging and biodistribution studies.

The ${ }^{153} \mathrm{Sm}$-loaded -PLGA nanoparticles had an average size of $281 \pm 6.3 \mathrm{~nm}$ and a PDI average of 0.22. The Zeta potential ranged between 5 and $20 \mathrm{mV}$. The $\left[{ }^{153} \mathrm{Sm}\right] \mathrm{Sm}_{2} \mathrm{O}_{3}$ loaded PLGA nanoparticles, orally administered were distributed to most organs at low levels, indicating that there was absorption of nanoparticles. While the IV injected $\left[{ }^{153} \mathrm{Sm}\right] \mathrm{Sm}_{2} \mathrm{O}_{3}$ loaded PLGA nanoparticles exhibited the highest localisation of nanoparticles in the spleen $(8.63 \% \mathrm{ID} / \mathrm{g})$ and liver $(3.07 \% \mathrm{ID} / \mathrm{g})$, confirming that nanoparticles are rapidly removed from the blood by the RES, leading to rapid uptake in the liver and spleen, From the biodistribution data obtained it is clear that polymeric nano scale delivery systems would be suitable for improving permeability and thus the bioavailability of therapeutic compounds.
\end{abstract}

Keywords: Biodistribution, In vivo, Imaging, Nanoparticles, PLGA, Samarium oxide 


\section{Graphical abstract}

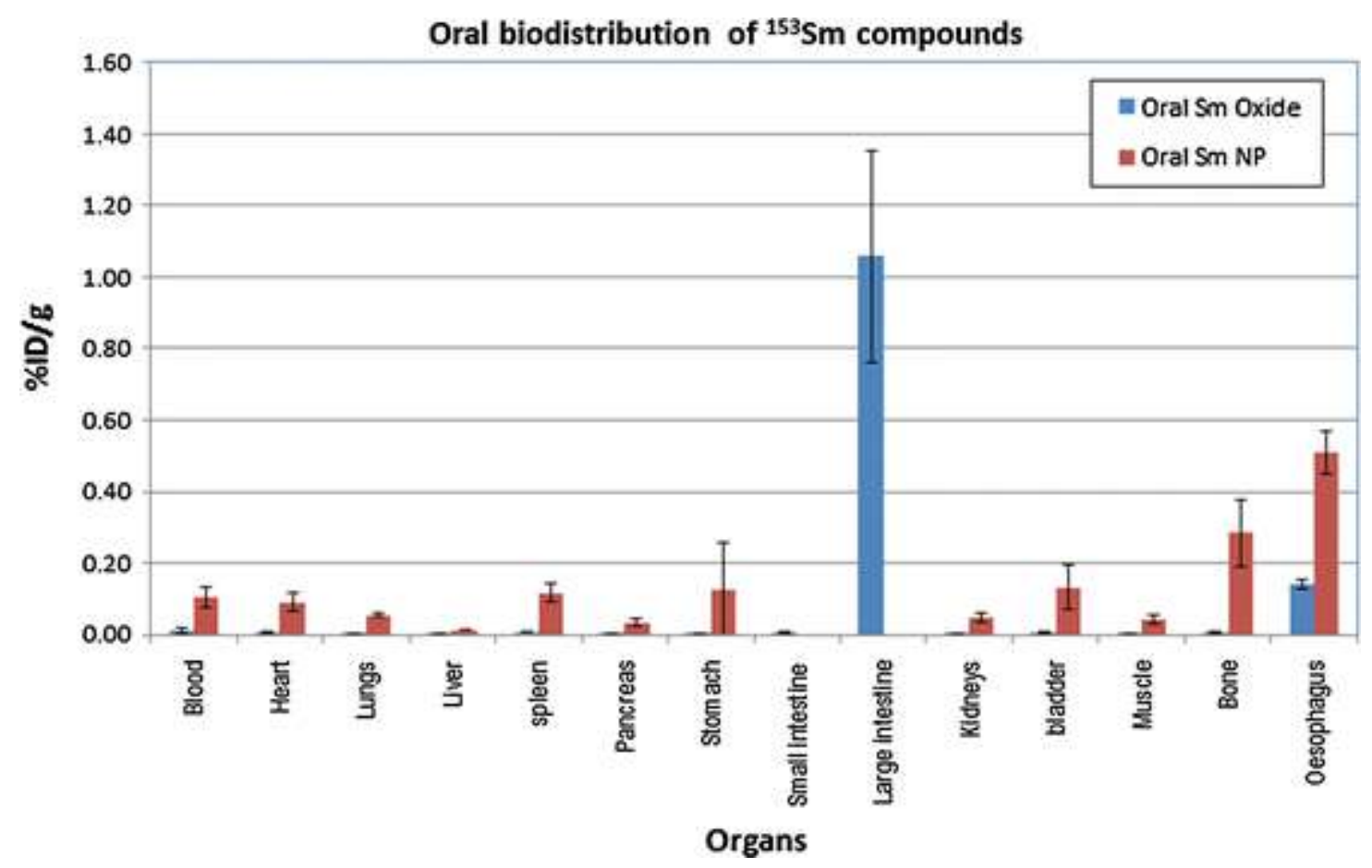

\section{Introduction}

Drug delivery has become a field of great interest to researchers mainly because delivering therapeutic agents or medicine to its site of action is one of the main limitations in pharmaceutical and biotechnology industries (Parveen et al., 2012). Drug delivery is a process of releasing a bioactive agent at a controlled rate at a target site (Orive et al., 2003; Parveen et al., 2012). A more targeted delivery system can help reduce or eliminate significant drug side effects by limiting the exposure of the drug to specific organs or tissue and reducing the drug concentration for treatment. Safe and targeted drug delivery could improve the performance of therapeutic drugs which are already in the market and influence the development and success of new therapeutic drugs.

Research in the field of nanotechnology has led to the development of nanoscale drug delivery systems, which focus on formulating biocompatible nanoscale delivery systems for drug delivery and imaging (Caruthers et al., 2007; Koo et al., 2005; Parveen et al., 2012). Nanoscale delivery systems can in this case be referred to as nanoparticles. Nanoparticles can be used to deliver drugs, recombinant proteins, vaccines and nucleotides (Parveen et al., 2012). Nanoparticulate delivery systems may include polymeric nanoparticles (Mohammad and Reineke, 2012; Parveen et al., 2012; Smola et al., 2008), solid lipid nanoparticles (SLN) (Parhi and Suresh, 2012), polymeric micelles (Parveen et al., 2012; Smola et al., 2008), liposomes (Ali et al., 2009; Koo et al., 2005) and dendrimers (Ali et al., 2009; Parveen et al., 2012). 
Enhanced in vivo drug biodistribution studies are important in improving the efficacy of these therapeutic drugs and in limiting their potential side effects. Monitoring the accumulation of a therapeutic formulation in specific organs or tissue in real-time can allow scientists to optimize the formulations to enhance their biodistribution properties. The most common method of tracking particle uptake or biodistribution involves the use of radiolabelled nanoparticles. The biodistribution data which is obtained gives an accurate representation of the tissues specifically targeted by a drug formulation and provide information on the main organs of clearance.

A powerful feature of nuclear molecular imaging is the evaluation of drug delivery systems in vivo. It is a technique which uses external detectors such as gamma $(\gamma)$ cameras to capture and form images from the radiation emitted by radiopharmaceuticals after they are administered orally or intravenously. These techniques allow drug molecules and delivery systems to be radiolabelled and their release, biodistribution and uptake may be visualized in vivo (Perkins and Frier, 2004, Venter et al. 2012). For example, by tagging a drug molecule, peptide, protein or a cell with a radiotracer, its site of release, distribution and metabolism can be studied. Imaging technology uses suitable $\gamma$ emitting radionuclides, commonly technetium-99m $\left({ }^{99 \mathrm{~m}} \mathrm{Tc}\right)$, indium-111 $\left({ }^{111} \mathrm{In}\right)$, iodine-123 $\left({ }^{123} \mathrm{I}\right)$ and samarium-153 $\left({ }^{153} \mathrm{Sm}\right)$ which may be imaged with a $\gamma$ camera. An advantage of these techniques is that the in vivo distribution and kinetics of a radiolabelled pharmaceutical formulation may be quantified; as a result a correlation between the observed pharmacological effects and the specific site of delivery may be made.

In this study, polymeric poly(D,L-lactide-co-glycolide) (PLGA) nanoparticles delivery systems will be discussed as well as their applications in imaging and biodistribution studies. The advantages of PLGA nanoparticles include the encapsulation of extremely hydrophobic and hydrophilic drugs as well as the controlled release of these actives. These delivery systems were intended to be radiolabelled by encapsulation with a radiotracer (samarium incorporated in the nanoformulation as samarium oxide). The conventional method of nanoparticle formulation involves encapsulating an anti-tuberculosis drug into the nanoparticle shell. To improve on the current inadequate control of tuberculosis (TB), polymeric anti-TB nanosized delivery systems containing anti-TB drugs which will enable entry, targeting, slow release and retention of the drugs in the cells for longer periods are being developed. This is done with the aim to hence reduce the dose frequency of the drug from daily intake to once a week. Thus these radiolabelled nanoparticle vectors will in this study be referred to as delivery systems because they do not contain any drug.

This particular study was done with the aim of this study was the coating of a nano-sized radioactive tracer (samarium oxide) by means of a biodegradable and biocompatible polymer aiming at visualising and tracking the fate as well as quantifying polymeric nanocarriers in vivo by using a single photon emission computed tomography (SPECT) gamma camera in Sprague Dawley Rats. These radiolabelled nanoparticles were not tracked by a radiolabel that adheres to the outer surface of the nanoparticle (which may be lost during in vivo studies) but non-radioactive $\mathrm{Sm}$ is entrapped in the PLGA nanoparticle in the same manner that a 
tuberculosis drug would be encapsulated and then activated. This is therefore a close resemblance to the ultimate use of the nanoparticles and provides translational data.

\section{Material and Methods}

\subsection{Materials}

Samarium oxide $\left(\mathrm{Sm}_{2} \mathrm{O}_{3}\right)$ 99\% enriched and $\mathrm{Sm}_{2} \mathrm{O}_{3}$ was obtained from Johnson Matthey (Johnson Matthey, Alfa, Ward Hill, MA,USA) and Sigma-Aldrich (Sigma-Aldrich, Germany) respectively. Polyvinyl alcohol (PVA), hydrolysed 87-89\%, Mw 13 000-23 000, Poly(D,L-lactide-co-glycolide) (PLGA), Mw 30 000-60 000, 50:50, Ethyl acetate, 99.5\% and Chitosan-De-Ac, Low viscous, 2-amino-2-deoxy-(1>4) B-D-glucopyranan were purchased from Sigma-Aldrich, Germany. Stearic acid, Mw $284.48 \mathrm{~g} / \mathrm{mol}$ was purchased from Merck (Darmstadt, Germany). Polyethylene glycol (PEG), Pluriol E 9000 was purchased from BASF (Ludwigshafen, Germany). Surfynol 104 PG 50 surfactant was purchased from Air Productions and Chemicals. Ketamine/ Xylazine was purchased from Phoenix Pharmaceutical (Baden-Wuerttemberg, Germany).

Both distilled and deionized water was used for the preparation of all solutions and emulsions.

\subsection{Methods}

In this study, polymeric PLGA nanoparticles were encapsulated with stable $\left[{ }^{152} \mathrm{Sm}\right] \mathrm{Sm}_{2} \mathrm{O}_{3}$. These were then activated to induce radioactive samarium- 153 oxide $\left(\left[{ }^{153} \mathrm{Sm} \mathrm{Sm}_{2} \mathrm{O}_{3}\right)\right.$ loaded PLGA nanoparticles via neutron activation in a nuclear reactor. The stable nanoparticles were characterized for size and Zeta potential using a Malvern Zetasizer Nano ZS and their morphology were established via scanning electron microscopy (SEM). After irradiation and sufficient decay this was repeated to ensure that neutron activation had no detrimental effect on the nanoparticles. Thereafter the radioactive $\left[{ }^{153} \mathrm{Sm}\right] \mathrm{Sm}_{2} \mathrm{O}_{3}$ loaded PLGA nanoparticles were orally and intravenously administered to rats in order to trace their uptake and biodistribution through imaging and biodistribution studies.

\subsubsection{Preparation of $\mathrm{Sm}_{2} \mathrm{O}_{3}$}

A small quantity of the raw samarium oxide $\left(\mathrm{Sm}_{2} \mathrm{O}_{3}\right)$ 99\% enriched (Johnson Matthey, Alfa, Ward Hill, MA, USA) powder was milled with a Stuart SHM1 high speed homogenizer (Bibby Scientific Ltd. Stone, Staffordshire, ST15 OSA, United Kingdom) to reach the desired nanometric size range (i.e. $<500 \mathrm{~nm}$ ) prior to emulsification. (Due to the cost of enriched material this procedure was first optimised with high purity natural $\mathrm{Sm}_{2} \mathrm{O}_{3}$ ). The milled $\mathrm{Sm}_{2} \mathrm{O}_{3}(50 \mathrm{mg})$ was dispersed in $2.5 \mathrm{ml}$ of an aqueous solution of $87-89 \%$ partially hydrolysed polyvinyl alcohol (PVA) (2\%w/v used as polymeric stabiliser) and the resulting dispersion was milled for $2 \mathrm{~min}$ at $35000 \mathrm{rpm}$ using the high speed homogenizer. Thereafter the coarse particles were separated by centrifuging at $3000 \mathrm{rpm}$ for $15 \mathrm{~min}$. The supernatant was collected to be added into the PLGA solution for the preparation of nanoparticles. 


\subsubsection{Preparation of $\mathrm{Sm}_{2} \mathrm{O}_{3}$ loaded PLGA nanoparticles}

PLGA coated nanoparticles were prepared with PLGA 50:50; Mw 30 000-60 000 purchased from Sigma-Aldrich, as the encapsulating polymer by using a modified double emulsion solvent evaporation technique (Semete et al., 2010; Lamprecht et al., 1999). Briefly, $100 \mathrm{mg}$ of PLGA was dissolved in $10 \mathrm{ml}$ of ethyl acetate containing stearic acid $0.2 \% \mathrm{w} / \mathrm{v}$ and one drop of surfynol 104 PG 50. A $2 \mathrm{ml}$ suspension of $\mathrm{Sm}_{2} \mathrm{O}_{3}$ supernatant was added into the dissolved PLGA solution. This mixture was emulsified by means of a Silverson L4R high speed homogenizer (Silverson Machines Limited, Buckinghamshire, United Kingdom) at $8000 \mathrm{rpm}$ for $10 \mathrm{~min}$ in an ice bucket. The resulting water-in-oil (w/o) emulsion was then transferred into a water phase of surface modifying excipients consisting of $15 \mathrm{ml}$ PVA $2 \% \mathrm{w} / \mathrm{v}, 5 \mathrm{ml}$ polyethylene glycol (PEG) $1 \% \mathrm{w} / \mathrm{v}, 10 \mathrm{ml}$ chitosan $0.3 \% \mathrm{w} / \mathrm{v}$ and $10 \mathrm{ml}$ lactose $5 \% \mathrm{w} / \mathrm{v}$ solution. The mixture was further emulsified using the homogenizer set at $8000 \mathrm{rpm}$ for $20 \mathrm{~min}$. The resulting water-in-oil-in-water (w/o/w) double emulsion was spray dried in a Buchi mini spray dryer (BUCHI Labortechnik AG, Flawil, Switzerland) at $96{ }^{\circ} \mathrm{C}$, an atomisation pressure of $7 \mathrm{bar}$, with a pump feeding rate of 2 and $100 \%$ aspiration to obtain a dry nanoparticle powder.

\subsubsection{Neutron activation of $\mathrm{Sm}_{2} \mathrm{O}_{3^{-}}$PLGA nanoparticles}

The nanoparticles were sent to the SAFARI-1 (South African Fundamental Atomic Research Installation) research reactor operated by the South African Nuclear Energy Corporation (Necsa), located at Pelindaba, South Africa, for neutron activation. The PLGA nanoparticle sample and a $\left[{ }^{152} \mathrm{Sm}\right] \mathrm{Sm}_{2} \mathrm{O}_{3}$ control were weighed out to $160 \mathrm{mg}$ and $10 \mathrm{mg}$ respectively and heat-sealed into individual polyethylene vials and packed into a polyethylene ampoule (commonly known as a rabbit). The ampoule was then delivered to the reactor core by a pneumatic transport system. The nanoparticles were then irradiated in a neutron flux of $2.8 \mathrm{x}$ $10^{14} \mathrm{~s}^{-1} \cdot \mathrm{cm}^{-2}$ for $60 \mathrm{~s}$ as set by preliminary test protocol to achieve a nominal radioactivity of $1 \mathrm{MBq}$ (megabecquerel) at $48 \mathrm{~h}$ after End of Irradiation (EoI). Two irradiation options are possible and were both successfully used; Cadmium (cadmium shielding is used to reduce the thermal neutron flux in the sample and mainly allow fast neutron activation reactions) and Non-cadmium (where the sample is exposed to the full neutron spectra). The nanoparticle samples were kept for at least $24 \mathrm{~h}$ after EoI to allow for the decay of unwanted irradiation by-products (Yeong et al., 2011(a) (b)). To allow for characterisation in non-radioactive laboratories as described below, the samples were kept for 30 days before release as nonradioactive material.

\subsection{Characterisation}

\subsubsection{Particle size and Zeta potential}

Particle size and size distribution indicated as the polydispersity index (PDI) were measured by dynamic laser scattering or photon correlation spectroscopy using a Malvern Zetasizer Nano ZS (Malvern Instruments, Worcestershire, United Kingdom). For each sample, 1-3 mg 
of $\mathrm{Sm}_{2} \mathrm{O}_{3}$-PLGA nanoparticles were suspended in distilled water, and then sonicated for a few minutes. Each sample was measured in triplicate.

The Zeta potential was determined using a Malvern Zetasizer Nano ZS (Malvern Instruments, Worcestershire, United Kingdom) at a $\mathrm{pH}$ of 6,8. The instrument calculates the nanoparticle net charge at the surface by determining the electrophoretic mobility using the Laser Doppler Velocity principle. For each sample, 1-3 mg of $\mathrm{Sm}_{2} \mathrm{O}_{3}$-PLGA nanoparticles were suspended in distilled water, then sonicated for a few minutes and introduced into a U-shape Zeta cell. Each sample was measured in triplicate to determine the Zeta potential.

\subsubsection{Surface morphology}

\subsubsection{Scanning Electron Microscopy}

SEM uses a focused beam of high-energy electrons to bombard a solid surface of a conductive sample to generate a variety of signals from the interaction volume. These electrons interact with the atoms of the sample producing signals which reveal visible information about the sample, relating to its morphology while size measurements of the structures are also possible. The morphology of the $\mathrm{Sm}_{2} \mathrm{O}_{3}$-PLGA nanoparticles was analysed using a scanning electron microscope (Carl Zeiss Microscopy, Oberkochen) operating at an accelerated voltage of $8 \mathrm{kV}$. The nanoparticle samples were initially thinly spread on a specimen holder using a spatula and adhered to the surface containing adhesive carbon tape. The nanoparticle samples were coated under argon atmosphere with an ultrathin $10 \mathrm{~nm}$ cobalt layer (Emitech K550 Super Coated; Emitech Ltd, South Stour Avenue Ashford, Kent, United Kingdom) to increase electrical conductivity. This is essential so as for samples to be imaged to prevent the accumulation of electrostatic charge build up at non-conductive areas on the sample surface. The stubs were then placed in a SEM high vacuum chamber for analysis. The nanoparticle images were analysed at different magnifications (5000 to 50 000x).

\subsubsection{Inductively Coupled Plasma Spectrometry}

To quantify the amount of metal in each nanoparticle sample, ICP-OES (Inductively Coupled Plasma-Optical Emission Spectrometry) was performed on a SPECTRO ARCOS (SPECTRO Analytical Instruments, Kleve, Germany). Each nanoparticle sample was diluted by acid digestion. The instrument was calibrated using the standard solutions. The samples were then run to generate a report. The optics are hermetically sealed and filled with argon, to achieve high optical transmission in the vacuum ultraviolet, allowing the determination of non-metals and interference free lines in this region. A wavelength region of between 130 and $770 \mathrm{~nm}$ can be simultaneously analysed, allowing capturing of a complete spectrum within $2 \mathrm{~s}$. 


\subsection{In vivo biodistribution studies}

\subsubsection{Animals}

The study was conducted in accordance with the South African National Standard for the Care and Use of Animals for Scientific Purpose. Ethical approval was sought and granted by the Ethics Committee for Research on Animals (ECRA), Tygerberg, Cape Town, South Africa (Approval No. 04/12). Healthy female, inbred Sprague Dawley Rats, weighing between 280 and $390 \mathrm{~g}$ were selected and housed under standard environmental conditions at an ambient temperature of $25{ }^{\circ} \mathrm{C}$. The rats were cared for and supplied with food and water ad libitum. The rats were allowed one week to acclimatize before the commencement of the study.

\subsubsection{Imaging study}

To obtain scintigraphic images of orally and intravenously administered radiolabelled particles, the rats were anaesthetised with Ketamine/ Xylazine $(90 / 10 \mathrm{mg} / \mathrm{kg})$. Only one rat per group was randomly selected for the imaging studies of $\mathrm{Sm}_{2} \mathrm{O}_{3}$ labelled PLGA (1) nanoparticles and $\mathrm{Sm}_{2} \mathrm{O}_{3}$ labelled PLGA (2) nanoparticles; oral and intravenous administration (IV). Immediately after receiving anaesthesia and radiolabelled nanoparticles, the rats were positioned under the gamma camera collimator (Infinia SPECT/CT, GE Healthcare, Salt Lake City, Utah and Lawrence, Massachusetts, USA) in ventral decubitas. The rats were injected IV with an average volume of $200 \mu 1$ or orally administered $500 \mu \mathrm{l}$ doses of radioactive nanoparticles with an activity of $7.85 \mathrm{MBq}$. Acquisition of dynamic images (120 s per frame) was recorded for the first $45 \mathrm{~min}$. Acquisition of static images was recorded at 1, 2, 4, 6, 24 and $48 \mathrm{~h}$ after administration for the $\mathrm{Sm}_{2} \mathrm{O}_{3}$ labelled PLGA nanoparticles. Regions of interest (ROIs) were drawn on the images of the liver, stomach, bladder, intestines and tail to obtain time activity curves from the dynamic imaging study. Data for the ROIs, which were decay corrected, were obtained from the static images. After acquisition of images, the rats were sacrificed via $100 \mathrm{mg}$ sodium pentobarbital/ $\mathrm{kg}$ intravenously. Several organs including the heart, lungs, liver, spleen, pancreas, stomach, intestines, kidneys, bladder, muscle, skin, bone, tail and blood were immediately removed after sacrificing the rats. Each organ was then weighed and the radioactivity was measured using a Capintec well type counter (Capintec, Inc, Ramsey, NJ, USA) as well as a MCA scintispec well counter.

\subsubsection{Biodistribution study}

To determine the biodistribution of the nanoparticles, radioactively labelled particles were orally and intravenously administered to rats. Twelve rats were assigned at random into four groups of three rats per group. These were further grouped into two groups per nanoparticle sample for the nanoparticle sample of $\mathrm{Sm}_{2} \mathrm{O}_{3}$ labelled PLGA nanoparticles and the $\mathrm{Sm}_{2} \mathrm{O}_{3}$ control, for both oral and IV administration. The ventral tail artery of all rats was catheterized under Ketamine/ Xylazine $(90 / 10 \mathrm{mg} / \mathrm{kg}$ ) anaesthesia immediately before the 
study. Additionally, lateral tail veins were catheterized for all the rats which were administered radiolabelled nanoparticle compounds intravenously. The catheter was then removed after intravenous administration. One dose of each radiolabelled nanoparticle compound was administered either via oral route $(500 \mu \mathrm{l}$ in saline) using a $22 \mathrm{G}$ feeding needle attached to a $1 \mathrm{ml}$ syringe or via intravenous route $(200 \mu \mathrm{l}$ in saline) through a catheterized tail vein with an activity of $0.23 \mathrm{MBq}$. Three rats were sacrificed at $24 \mathrm{~h}$ via 100 $\mathrm{mg}$ sodium pentobarbital/ $\mathrm{kg}$ intravenously. The heart, lungs, liver, spleen, pancreas, stomach, intestines, kidneys, bladder, muscle, skin, bone, tail and blood were immediately removed after killing the rats. Each organ was then contained in a test tube, weighed and the radioactivity was measured using a Capintec well type counter (Capintec, Inc, Ramsey, NJ, USA) as well as a MCA scintispec well counter. Three dilutions of the radioactive solution were made to correlate counts with activity measured in MBq.

\section{Results and discussion}

\subsection{Neutron activation of $\mathrm{Sm}_{2} \mathrm{O}_{3}-\mathrm{PLGA}$ nanoparticles}

The $\left[{ }^{152} \mathrm{Sm}\right] \mathrm{Sm}_{2} \mathrm{O}_{3}$-PLGA nanoparticle test compounds were irradiated via neutron activation and activity reported in $\mathrm{MBq}$. The activity measured in two $\left[{ }^{153} \mathrm{Sm}\right] \mathrm{Sm}_{2} \mathrm{O}_{3}$-PLGA nanoparticle samples after neutron activation was 11 (sample 1) and $1.2 \mathrm{MBq}$ (sample 2). The radioactivity levels differed greatly due to the irradiation method employed (noncadmium and cadmium), in which the non-cadmium method (Sm loaded nanoparticles exposed to the full neutron spectra) yielded higher radioactivity. The mean activity at time of administration was 8.90 (sample 1) and $0.90 \mathrm{MBq}$ (sample 2) respectively. The activity of the $\left[{ }^{153} \mathrm{Sm}\right] \mathrm{Sm}_{2} \mathrm{O}_{3}$-PLGA nanoparticles' injected doses were 4.49 and $0.49 \mathrm{MBq}$ each in a $200 \mu 1$ saline suspension for the imaging and biodistribution (only) study respectively.

\subsection{Characterisation}

\subsubsection{Particle size and Zeta potential}

The characterisation of nanoparticles is required to control the quality of the manufactured nanoparticles. The particle size and surface charge are some of the parameters which need to be assessed because of their direct impact on the stability and release kinetics of the radionuclides which have been incorporated (Parhi and Suresh, 2012).

Three homogenization cycles of 800 bars were used and it was found that all nanoparticle formulations had a particle size less than $500 \mathrm{~nm}$. Various parameters were optimized to obtain an average particle size ranging between 250 and $300 \mathrm{~nm}$, with an average polydispersity index $\leq 0.3$ after spray drying. The particles had a Zeta potential ranging between 5 and $20 \mathrm{mV}$. 
There were no significant differences between the two $\left[{ }^{153} \mathrm{Sm}\right] \mathrm{Sm}_{2} \mathrm{O}_{3}$-PLGA nanoparticles, henceforth simplified as $\mathrm{Sm}_{2} \mathrm{O}_{3}$-PLGA nanoparticles in terms of size, size dispersion and surface charge. The nanoparticles portrayed a size range below $300 \mathrm{~nm}$, an average size distribution of 0.2 and a Zeta potential between 5 and $8 \mathrm{mV}$

(Table 1). The bigger the Zeta potential of the suspension, the more likely it is to be stable because the charged particles repel each other and therefore overcome the natural tendency to aggregate (Parhi and Suresh, 2012).

Table 1: Size and Zeta potential of $\mathrm{Sm}_{2} \mathrm{O}_{3}$ loaded PLGA nanoparticles

\begin{tabular}{llll} 
Sample & Size $(\mathbf{n m})$ & PDI & Zeta potential $(\mathbf{m V})$ \\
& & & \\
\hline 1 (before irradiation) & $270.70 \pm 6.80$ & $0.21 \pm 0.18$ & $7.45 \pm 0.49$ \\
2 (before irradiation) & $292.10 \pm 5.80$ & $0.24 \pm 0.02$ & $5.40 \pm 1.01$ \\
1 (after irradiation) & $286.70 \pm 8.56$ & $0.23 \pm 0.01$ & $17.00 \pm 0.36$ \\
2 (after irradiation) & $333.90 \pm 181.4$ & $0.59 \pm 0.13$ & $5.52 \pm 2.97$ \\
\hline
\end{tabular}

PDI: polydispersity index, nm: nanometres, $\mathrm{mV}$ : millivolts

The $\mathrm{Sm}_{2} \mathrm{O}_{3}$-PLGA nanoparticles were irradiated via neutron activation to achieve a nominal radioactivity of $1 \mathrm{MBq}$ at $48 \mathrm{~h}$ after End of Irradiation (EoI) (Yeong et al., 2011(a)). To allow for further size and morphological characterisation in non-radioactive laboratories, the sample was kept for 30 days before released as non-radioactive material. The nanoparticles were larger in size, with an increased PDI and Zeta potential after the irradiation process (Table 1). As depicted by size analysis, the nanoparticles could have aggregated and increased in size as a result of the increase in Zeta potential during the irradiation process. It is accepted that Zeta potentials more than $30 \mathrm{mV}$ are sufficient for good electrostatic stabilization (Parhi and Suresh, 2012). In order, to minimise the effect of aggregation after irradiation, nanoparticles were sonicated /vortexed before administration.

\subsection{Surface morphology}

\subsection{Scanning electron microscopy}

SEM images of the non-irradiated $\mathrm{Sm}_{2} \mathrm{O}_{3}$-PLGA nanoparticles showed spherical particles with a smooth surface. The particle sizes ranged between 200 and $600 \mathrm{~nm}$, with a few particles in the $1 \mu \mathrm{m}$ range. These images give a visual profile of the size and size distribution results obtained from the dynamic laser scattering using a Malvern Zetasizer Nano ZS. 


\subsection{Inductively Coupled Plasma Spectrometry}

To quantify the amount of metal in each nanoparticle sample, ICP-OES (Inductively Coupled Plasma-Optical Emission Spectrometry) was performed. PLGA nanoparticles containing $\mathrm{Sm}_{2} \mathrm{O}_{3}$ contained an average amount of $20621 \mathrm{mg} / \mathrm{kg}(2 \%)$ samarium-152 (Table 3). ICP confirmed the presence of the tracer elements but does not however inform if the tracers are encapsulated within the nanoparticles or on the surface.

Table 2: ICP spectrometry results $\mathrm{Sm}_{2} \mathrm{O}_{3}$ loaded PLGA nanoparticles before neutron activation

\section{Sample Result $(\mathrm{mg} / \mathrm{kg}) \quad$ Percentage (\%)}

\begin{tabular}{lll}
\hline 1 & 20918 & 2 \\
2 & 20324 & 2 \\
\end{tabular}

\subsection{In vivo biodistribution studies}

\subsubsection{Imaging and Biodistribution assays}

\subsubsection{Scintigraphic images: $\mathrm{Sm}_{2} \mathrm{O}_{3}$-PLGA nanoparticles}

The imaging procedures for $\left[{ }^{153} \mathrm{Sm}\right] \mathrm{Sm}_{2} \mathrm{O}_{3}$-PLGA nanoparticles were employed on four rats. The test compounds included two $\left[{ }^{153} \mathrm{Sm}\right] \mathrm{Sm}_{2} \mathrm{O}_{3}$ radiolabelled PLGA nanoparticles which were each administered one orally and one intravenously in the rats for imaging purposes. Imaging was used as a screening tool to evaluate the biodistribution over time and determine the (time) end point for the biodistribution only studies. The $\mathrm{Sm}_{2} \mathrm{O}_{3}$-PLGA nanoparticle formulation used for scintigraphic images had a nanoparticle size of $270 \mathrm{~nm}$, a size distribution of 0.21 and a Zeta potential of $7.45 \mathrm{mV}$. The activity measured after neutron activation was $11 \mathrm{MBq}$ per sample. The mean activity at time of administration was 8.9 $\mathrm{MBq}$. The gamma spectroscopic analysis demonstrated that the main photopeak measured from the organs after imaging was in the expected energy region of 94 to $125 \mathrm{keV}$. Additional peaks were detected in the region of 97 and $103 \mathrm{keV}$, which were recognized as the associated gamma energies emitted from the ${ }^{153} \mathrm{Sm}$ (Yeong et al., 2012).

Once the $\mathrm{Sm}_{2} \mathrm{O}_{3}$-PLGA nanoparticles were administered, only a trace amount of the nanoparticles was trapped in the site of administration (oesophagus or tail). After $24 \mathrm{~h}$ and $48 \mathrm{~h}$ (Figure 1) the nanoparticles had optimally distributed to other organs, but remained prominent in the stomach, liver and small intestines. The stomach was the main organ of particle absorption for orally administered $\mathrm{Sm}_{2} \mathrm{O}_{3}$-PLGA nanoparticles (Figure 2). The liver was the main organ of particle deposition following intravenous administration. In both 
circumstances, the $\mathrm{Sm}_{2} \mathrm{O}_{3}$-PLGA nanoparticles distributed from the latter organs to the intestines.
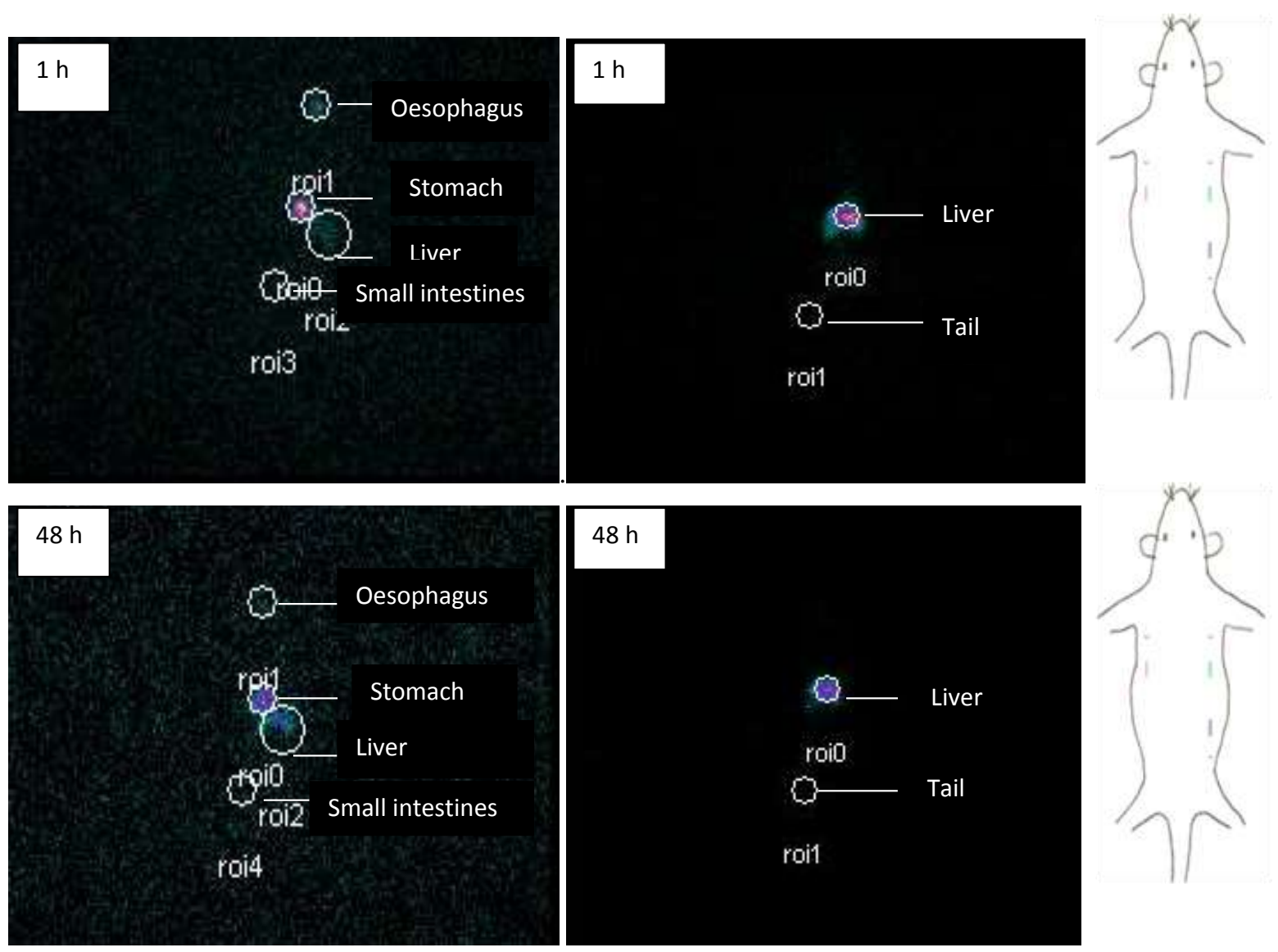

A

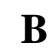

Figure 1: Static scintigraphic images of orally (A) and intravenously (B) administered radioactive $\mathrm{Sm}_{2} \mathrm{O}_{3}$-PLGA nanoparticles at 1 and $48 \mathrm{~h}$. 



A

B

Figure 2: Static scintigraphic images of orally (A) and intravenously (B) administered radioactive $\mathrm{Sm}_{2} \mathrm{O}_{3}$-PLGA nanoparticles at 1 and $24 \mathrm{~h}$

\subsubsection{Biodistribution of $\mathrm{Sm}_{2} \mathrm{O}_{3}-P L G A$ nanoparticles}

For the $\mathrm{Sm}_{2} \mathrm{O}_{3}$ control compound, the large intestine $(1.06 \% \mathrm{ID} / \mathrm{g})$ was the organ with the highest amount of deposition, and the oesophagus (site of administration) $(0.14 \% \mathrm{ID} / \mathrm{g}$ ) (Figure 3). $\mathrm{Sm}_{2} \mathrm{O}_{3}$ was most probably not absorbed in the stomach and small intestines; therefore it accumulated in the large intestine and eliminated via the large intestines. More than $90 \%$ of the $\mathrm{Sm}_{2} \mathrm{O}_{3}$ were excreted in $48 \mathrm{~h}$. 




Figure 3: The biodistribution of orally administered $\mathrm{Sm}_{2} \mathrm{O}_{3}$ - PLGA nanoparticles and $\mathrm{Sm}_{2} \mathrm{O}_{3}$ after $24 \mathrm{~h}(\mathrm{n}=3)$.

The $\left[{ }^{153} \mathrm{Sm}\right] \mathrm{Sm}_{2} \mathrm{O}_{3}$-PLGA nanoparticles were distributed to most organs although at low levels (Figure 3), which suggested that there was absorption of nanoparticles. From this distribution, we can assume that the nanoparticles facilitated the transport or distribution of the ${ }^{153} \mathrm{Sm}$ into the various organs. The nanoparticles also showed slower distribution and longer residence time in the organs than the $\mathrm{Sm}_{2} \mathrm{O}_{3}$ control (Mohammad and Reineke, 2013; Semete et al., 2010). This could possibly be due to the positive charge of the particles which cause adhesion to mucus layers in the GI tract (Semete et al., 2010). Nanoparticles enhance absorption of the encapsulated compound $\left({ }^{153} \mathrm{Sm}\right)$. The $\mathrm{Sm}_{2} \mathrm{O}_{3}$-PLGA nanoparticles were mostly deposited the oesophagus $(0.51 \% \mathrm{ID} / \mathrm{g})$, bone $(0.28 \% \mathrm{ID} / \mathrm{g})$ and bladder $(0.13 \% \mathrm{ID} / \mathrm{g})$. Both the control and the nanoparticle samarium compounds were detected oesophagus (site of administration) and absent in the small intestines.

Studying the in vivo distribution of the intravenously injected compounds showed that the highest trace amount of the control compound was reported in the tail (the site of injection) $(0.63 \% \mathrm{ID} / \mathrm{g})$ and the liver $(0.12 \% \mathrm{ID} / \mathrm{g})$. The stomach, spleen, lungs, small intestines, large intestines and kidneys presented no traces of ${ }^{153} \mathrm{Sm}_{2} \mathrm{O}_{3}$ (Figure 4). PLGA nanoparticles accumulated in the liver and spleen (Figure 4 and 5), which is the reported and the expected fate of intravenously administered PLGA nanoparticles (Semete et al., 2010). Nanoparticles 
with hydrophobic surfaces are rapidly sequestered from the circulation by organs of the mononuclear phagocyte system (i.e. liver, spleen, lungs) (Mohammad and Reineke, 2013). The $\mathrm{Sm}_{2} \mathrm{O}_{3}$-PLGA nanoparticles were deposited mostly in the spleen $(8.63 \% \mathrm{ID} / \mathrm{g})$ followed by the liver $(3.07 \% \mathrm{ID} / \mathrm{g})$. From this data, we know that the radiotracer was encapsulated because the encapsulated compound followed the distribution of PLGA nanoparticles. Reports show that particles bigger than $250 \mathrm{~nm}$ deposit and accumulate to a greater extent in the liver and spleen than smaller ones (Mohammad and Reineke, 2013). The bladder $(0.19 \% \mathrm{ID} / \mathrm{g})$, blood and kidneys $(0.15 \% \mathrm{ID} / \mathrm{g})$ and lungs $(0.13 \% \mathrm{ID} / \mathrm{g})$ also showed trace amounts of deposited $\mathrm{Sm}_{2} \mathrm{O}_{3}$-PLGA nanoparticles. Due to the $\mathrm{Sm}_{2} \mathrm{O}_{3}$ having a faster clearance than the $\mathrm{Sm}_{2} \mathrm{O}_{3}$-PLGA nanoparticles; it can be assumed that PLGA nanoparticles as a delivery system slow down clearance of ${ }^{153} \mathrm{Sm}$ and assisted with the biodistribution in various organs. In both ${ }^{153} \mathrm{Sm}$ compounds, injected doses per gram of organ of less than $0.04 \% \mathrm{ID} / \mathrm{g}$ were reported in the heart, muscles, pancreas, stomach, small and large intestines.

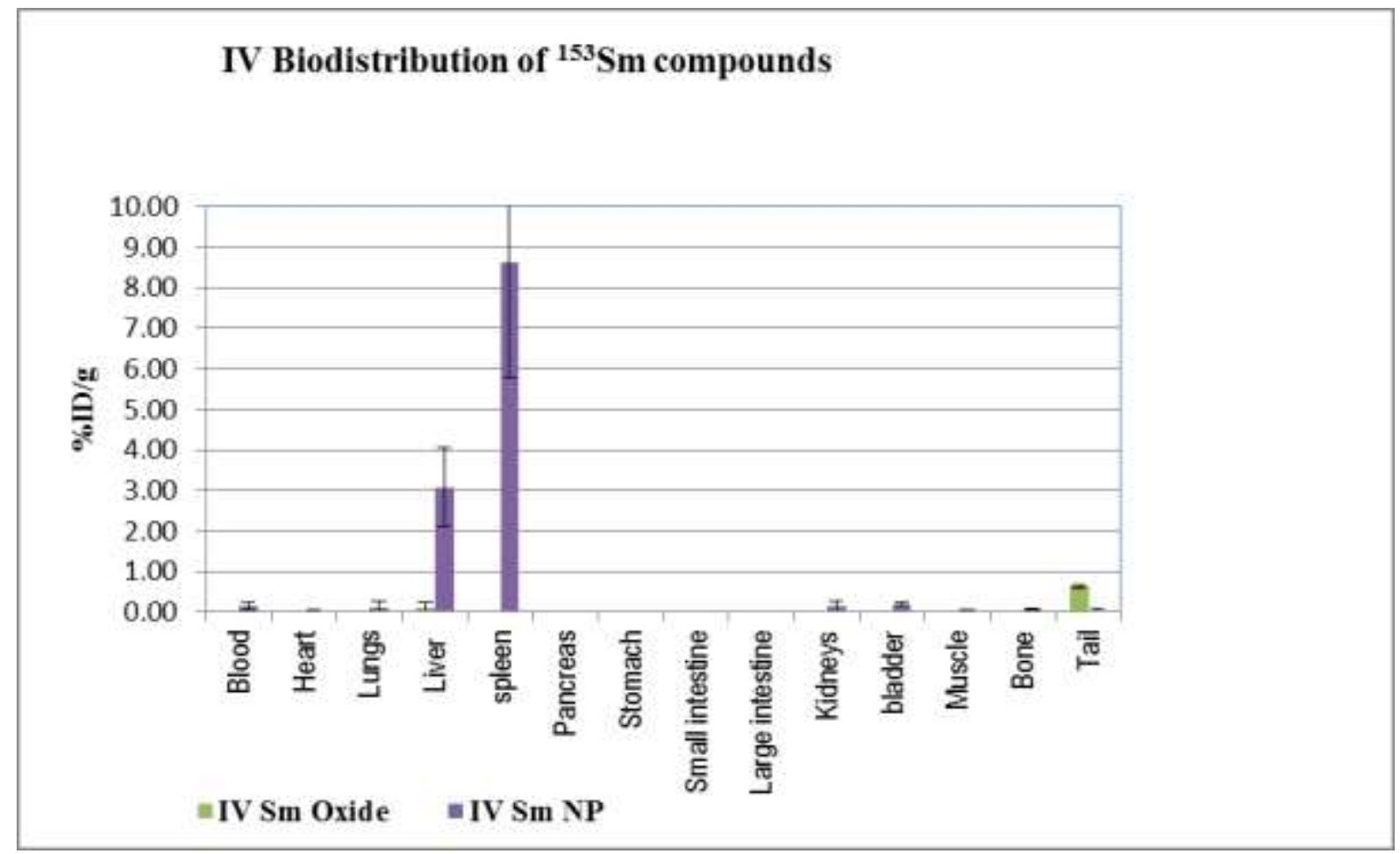

Figure 4: The in vivo biodistribution of intravenously injected $\mathrm{Sm}_{2} \mathrm{O}_{3}$ - PLGA nanoparticles and $\mathrm{Sm}_{2} \mathrm{O}_{3}$ after $24 \mathrm{~h}$. $(\mathrm{n}=3)$ 


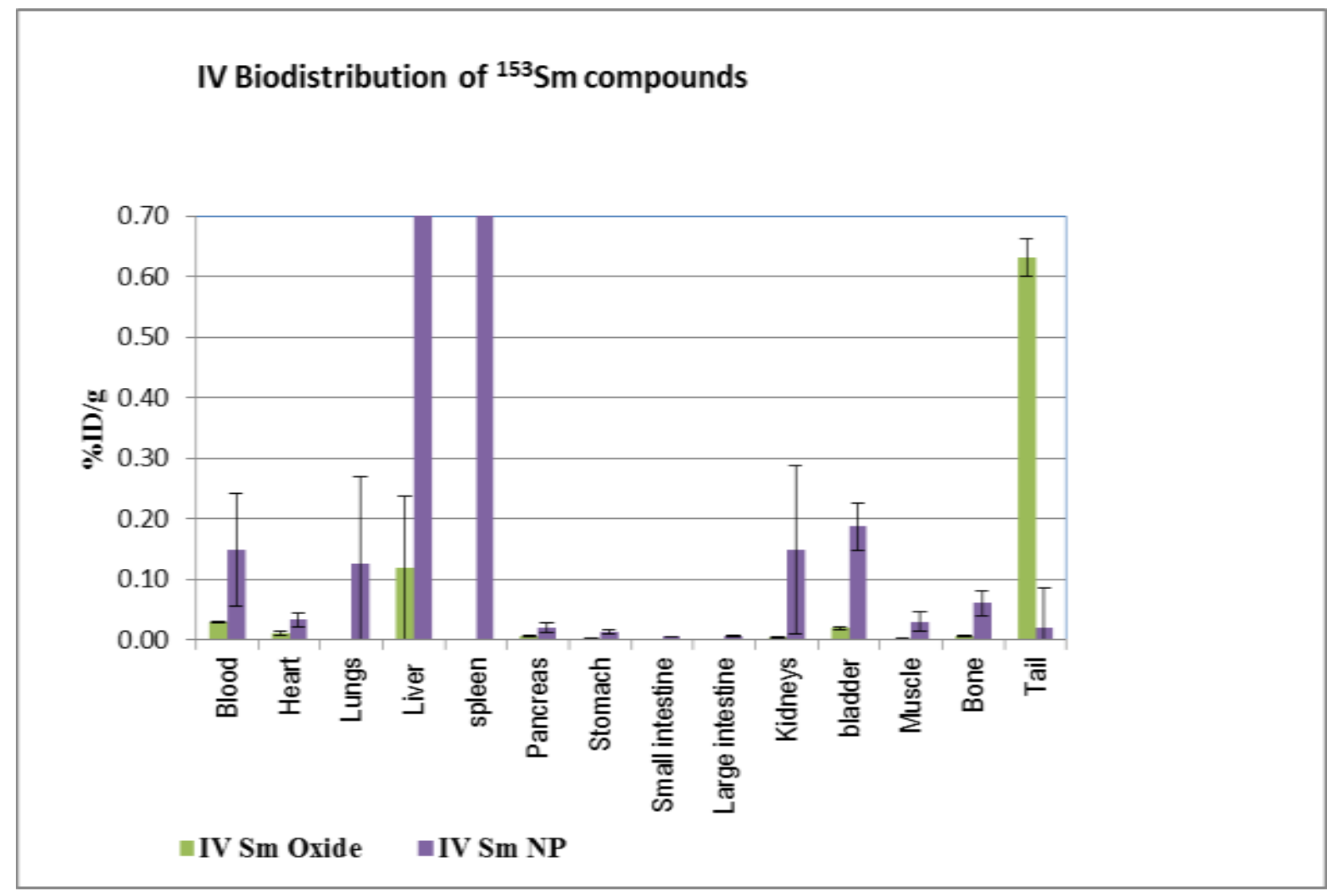

Figure 5: The in vivo biodistribution of intravenously injected $\mathrm{Sm}_{2} \mathrm{O}_{3}$ - PLGA nanoparticles and $\mathrm{Sm}_{2} \mathrm{O}_{3}$ after $24 \mathrm{~h}$ - adjusted y-axis. $(\mathrm{n}=3)$

\section{Conclusion}

The encapsulation of samarium oxide- $153\left[{ }^{153} \mathrm{Sm}\right] \mathrm{Sm}_{2} \mathrm{O}_{3}$ into PLGA was successfully carried out followed by brief activation of the $\mathrm{Sm}_{2} \mathrm{O}_{3}$ loaded PLGA nanoparticles in a nuclear reactor. The irradiation time of the $\mathrm{Sm}_{2} \mathrm{O}_{3}$ loaded PLGA nanoparticles in the nuclear reactor had no negative effect on the physicochemical properties of the nanoparticles and yielded enough radioactivity for scintigraphic imaging. This work demonstrated succesful radiolabelling and the the ability to image and quantify the distribution of the encapsulated radioactive $\mathrm{Sm}_{2} \mathrm{O}_{3}$ in nano scale delivery systems in each organ with the long-term hope of optimizing treatment protocols with improved drug bioavailability and reduced toxicity to normal tissues.

Nanoparticles aim to provide sustained release of the drug or encapsulated compound from the matrix or shell, which is supported by our results. The encapsulation of $\left[{ }^{153} \mathrm{Sm}\right] \mathrm{Sm}_{2} \mathrm{O}_{3}$ in PGLA nanoparticles changed the biodistribution ${ }^{153} \mathrm{Sm}_{2} \mathrm{O}_{3}$, both for orally and intravenously administered. PLGA nanoparticles also appear to enhance the intestinal absorption of ${ }^{153} \mathrm{Sm}$ in vivo as orally administered $\left[{ }^{153} \mathrm{Sm}\right] \mathrm{Sm}_{2} \mathrm{O}_{3}$ loaded in PLGA nanoparticles distributed to most organs. 
Although many concerns exist regarding the safety of nanoparticles in general, current progress from our study demonstrates evidence from the biodistribution studies, the inductively coupled spectrometry and scintigraphic imaging results that PLGA nanoparticles are safe to use as delivery systems when administered both orally and intravenously in the mentioned doses,. This does not, however, rule out that more studies need to be conducted to evaluate the safety or toxicity of nanoscale PLGA and other polymeric delivery systems. From the biodistribution data obtained it is clear that polymeric nanoscale delivery systems would be suitable for improving permeability and thus the bioavailability of therapeutic compounds. With this approach, delivery of poorly permeable and soluble drugs can be enhanced with effective drug delivery systems.

\section{Acknowledgements}

The authors would like to thank the Nuclear Technologies in Medicine and the Biosciences Initiative (NTeMBI), a national technology platform developed and managed by the South African Nuclear Energy Corporation (Necsa) and funded by the Department of Science and Technology. A special thanks to Cindy Els and Delene van Wyk at the Steve Biko Academic Hospital for assisting with the scintigraphic imaging.

\section{References}

M.M. Ali, B. Yoo and M.D. Pagel, Tracking the relative in vivo pharmacokinetics of nanoparticles with PARACEST MRI, Molecular Pharmaceutics 6(5) (2009) 1409-1416.

S.D. Caruthers, S.A. Wickline and G.M. Lanza, Nanotechnological applications in medicine, Current Opinion in Biotechnology 18(1) (2007) 26-30.

O.M. Koo, I. Rubinstein and H. Onyuksel, Role of nanotechnology in targeted drug delivery and imaging: a concise review, Nanomedicine: Nanotechnology, Biology and Medicine 1 (2005) 193-212.

A. Lambrecht, N. Ubrich, M. Hombreiro Perez, C.M. Lehr, J. Hoffman and P. Maincent, Biodegradable monodispersed nanoparticles prepared by pressure homogenizationemulsification, International Journal of Pharmaceutics 184(1) (1999) 97-105.

A.K. Mohammed and J.J. Reineke, Quantitative detection of PLGA nanoparticles degradation in tissues following intravenous administration, Molecular Pharmaceutics 10(6) (2013) 2183-2189.

G. Orive, R.M. Hernandez, A. Rodriguez Gascon, A. Dominguez-Gil and Z.J. Pedra, Drug delivery in biotechnology: present and future, Current Opinion in Biotechnology 14 (2003) 659-664. 
S. Parveen, R. Misra and S.K. Sahoo, Nanoparticles: a boon to drug delivery, therapeutics, diagnostics and imaging, Nanomedicine: Nanotechnology, Biology and Medicine 8 (2012) 147-166.

A.C. Perkins and M. Frier, Radionuclide imaging in drug development, Current Pharmaceutical Design 10 (2004) 2907-2921.

B. Semete, L. Booysen, Y. Lemmer, L. Kalombo, J. Venter, L. Katata, J. Verschoor and H. Swai, In vivo evaluation of the biodistribution and safety of PLGA nanoparticles as drug delivery systems, Nanomedicine: Nanotechnology, Biology and Medicine 6 (2010) 662-671.

M. Smola, T. Vandamme and A. Sokolowski, Nanocarriers aspulmonary drug delivery systems to treat and to diagnose respiratory and non respiratory diseases, International Journal of Nanomedicine 3(1) (2008) 1-19.

L. Venter, D. Jansen, J. Wagener, P.B. Fourie, J.R. Zeevaart, Characterization of radiolabelled dry powder leucine, a constituent of inhalable capreomycin, Drug delivery letters 2 (2012) 155-161.

A.V. Vergoni, G. Tosi, R. Tacchi, M.A. VAndelli, A. Bertolini and L. Costantino, Nanoparticles as drug delivery agents specific for CNS: in vivo biodistribution, Nanomedicine: Nanotechnology, Biology and Medicine 5 (2009) 369-377.

C.H. Yeong, B.J.J. Abdullah, K.H. Ng, L.Y. Chung, K.L. Goh, S.A. Sarji and A.C. Perkins, Neutron activated ${ }^{153} \mathrm{Sm}$-ion-exchange resin as a tracer for gastrointestinal Scintigraphy, Nuclear Medicine Communication 32(12) (2011(a)) 1256-1260.

C.H. Yeong, P.E. Blackshaw, K.H. Ng, B.J.J. Abdullah, M. Blaauw, R.J. Dansereau and A.C. Perkins, Reproductivity of neutron activated Sm-153 oral dose formulations intended for human administration, Applied Radiation and Isotopes 69(9) (2011(b)) 1181-1184.

C.H. Yeong, B.J.J. Abdullah, K.H. Ng, L.Y. Chung, K.L. Goh, S.A. Sarji and A.C. Perkins, Production and first use of ${ }^{153} \mathrm{SmCl}_{3}$-ion exchange resin capsule formulation for assessing gastrointestinal motility, Applied Radiation and Isotopes 70 (2012) 450-455. 